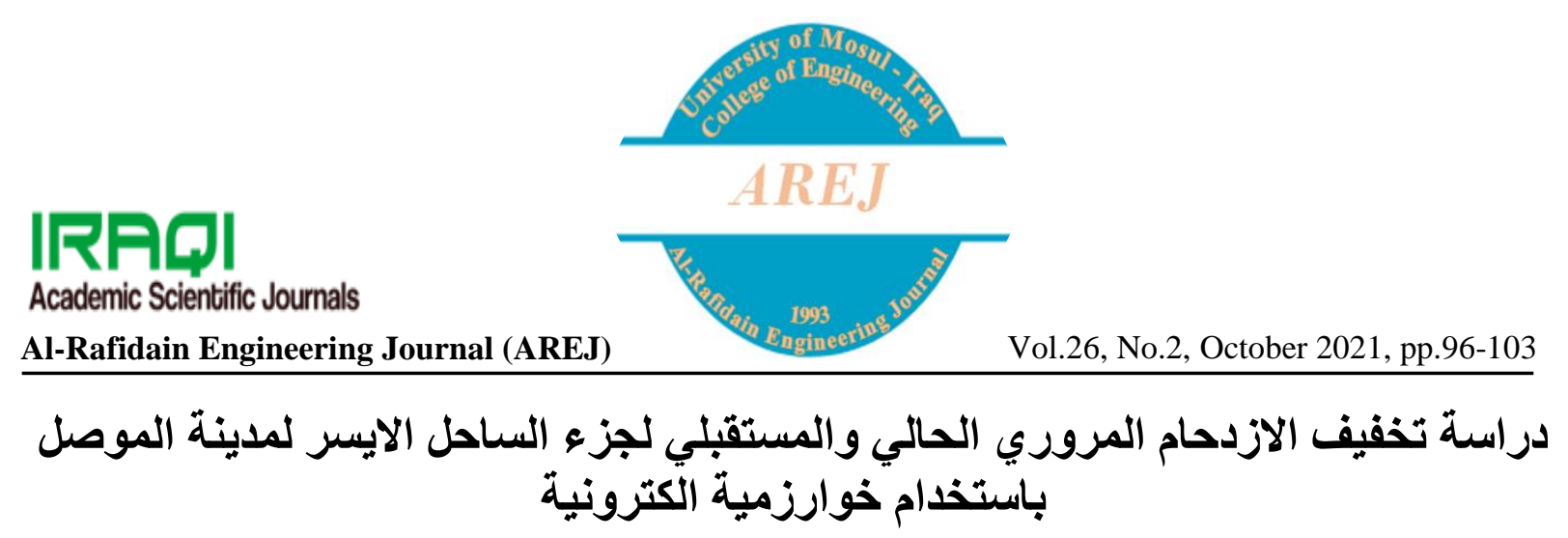

عبد الخالق مال الله محمد

abdelkhaleq@uomosul.edu.iq

تاريخ القبول: 11/3/2021

\author{
مهند رضنوان محمد عسكر
}

civilmohanadtraf2018@gmail.com

جامعة الموصل ـ كلية الهندسة ـ قسم الهندسة المدنية
تاريخ الاستلام: 7/10/2020 ت

يتنبر تعبين حركة المرور عملية مهمة جدا من عمليات تخطبط النقل الحضري:تم تطبيق خوارزمبية تعبين حركة المرور والتي تم تطوبير ها باستخدام عدة

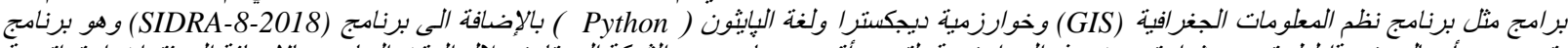

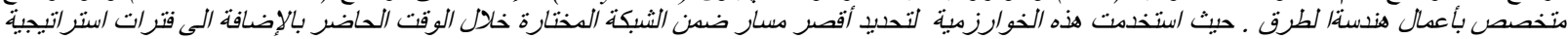

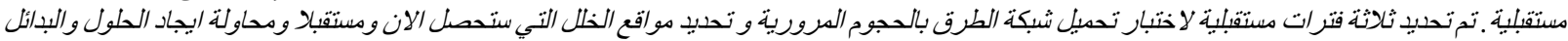

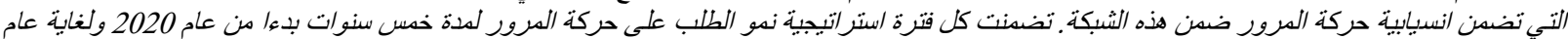

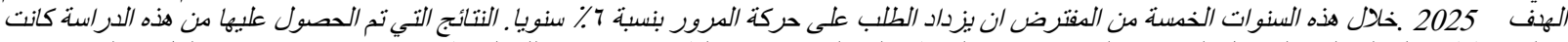

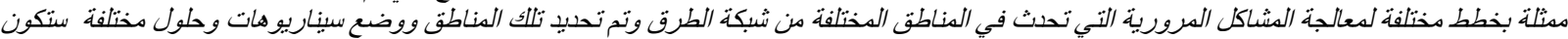
مطلوبة لتنظيم حركة المرور للفترات الاستر اتيجية.

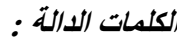

التوزيع المروري، مدينة الموحل، مستوى الخدمة ، السعة المحددة.

This is an open access article under the CC BY 4.0 license (http://creativecommons.org/licenses/by/4.0/).

https://rengj.mosuljournals.com

3- وضع برنامج يمكن استخدامه لأية مدينة تعاني من مشاكل الازدحامات المرورية إنعن

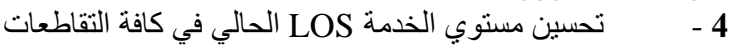

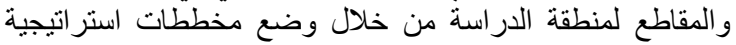

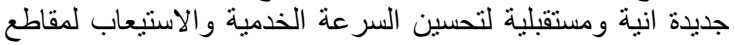
وتقاطعات شبكة الطرق المختلفة في ايسر الموصن الفئل

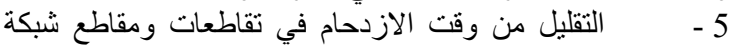

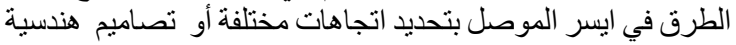

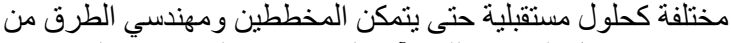

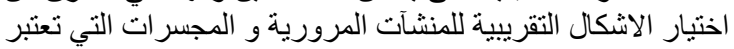

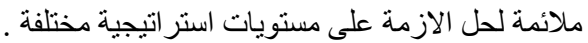
6 -تحديد اولويات قصبرة المدى لتنفيذ تحسينات في مر افق منظومة النقل. 7 -توفير البيانات و المعلومات اللازمة لعملية التخطيط بصورة عامة.

8 -توفير خطة فعالة لتوزيع الحجوم المرورية على شبكة الطرق.

3. منطقة الدراسة

تم اختيار جزء معين من الجانب الأيسر كمنطقة دراسة حيث

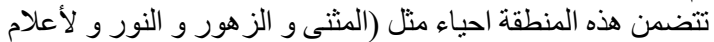

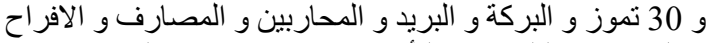

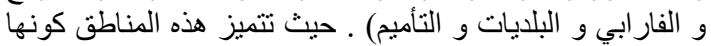

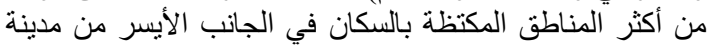

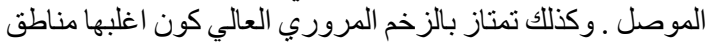

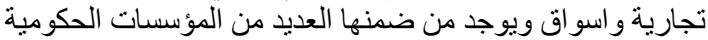
و التربوية ـوكذلك منطقة الدراسة محاذية للمنافذ باتجاه مدينتي المني

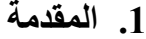

تتضمن عملية النقل الحضري التي تعتمد على التنبؤ

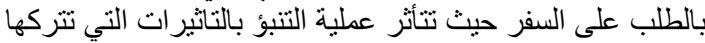

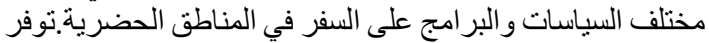

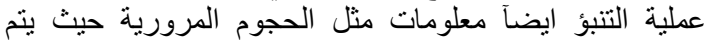

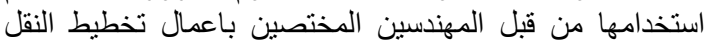

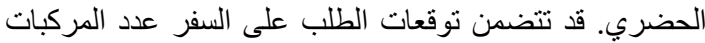

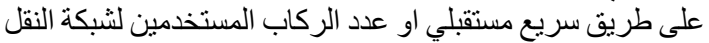

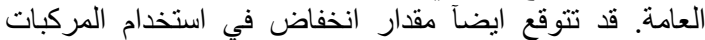

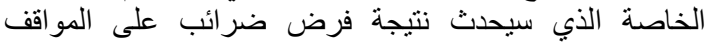

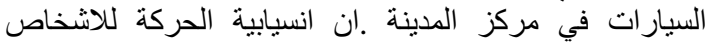

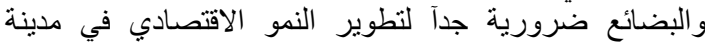

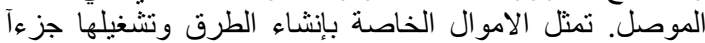

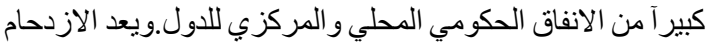

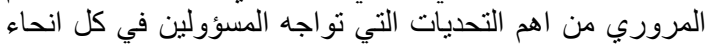

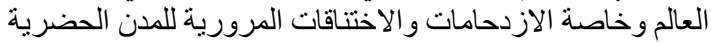

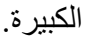

2. أهداف الدراسة

1 - تحسين الاستيعاب المروري في شبكة الطرق الحالية

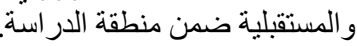

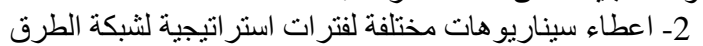

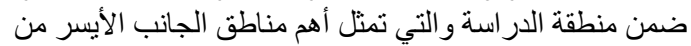
مدينة الموصل لتوفير أفضل انسيابية لحركة المرور. 
الزمنية للتنبؤ في هذه الدراسة تمتد الى خمسة أعوام كفترة

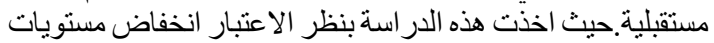

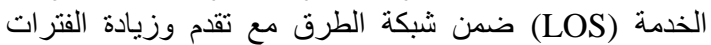

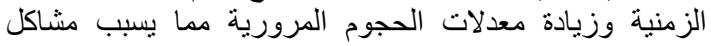
مرورية في مناطق معينة ضمن الثبكة ومحاولة اليجاد الحلول التهل لهذه المشاكل ضدمن كل فترة مستقبلية.

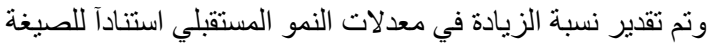

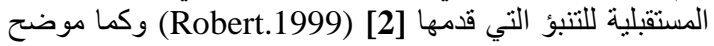

$\mathrm{Qf}=\mathrm{Qe}(1+\mathrm{r})$

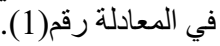

Qf: حجم الحركة المرورية لسنة الهدف (1)

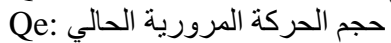

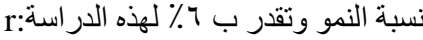

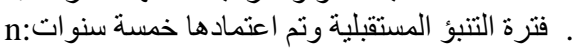

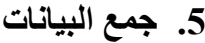

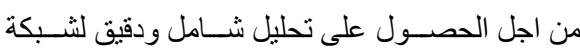

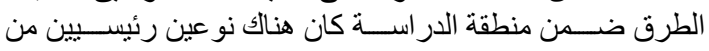

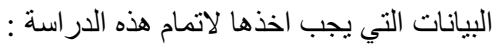

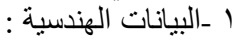

تضمنت هذه المرحلة اخذ كافة البيانات الهندسية للمقاطع المرورية

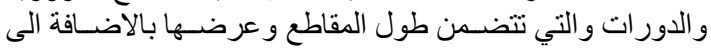
عدد الممر ات فيها و الميول الافقية و العمودية ولئ واتجاه

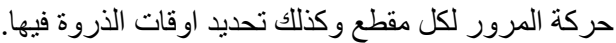

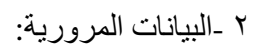

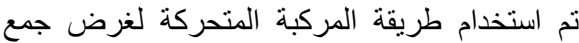

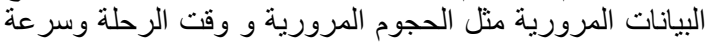

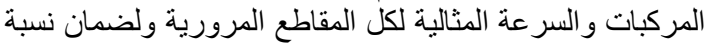

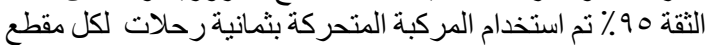

وفقال. [3 [3 (Garber and Lester,H,2009) وكذلك تم حساب الحجوم المرورية وزمن عبور المركبة وقيمة

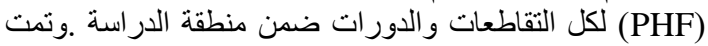

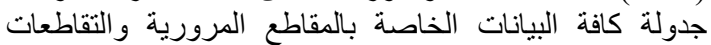

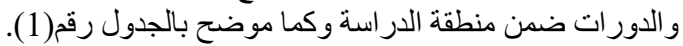
جدول رقم (1) : يوضح جدولة البيانات

\begin{tabular}{|c|c|c|c|c|c|c|c|c|c|c|c|c|c|c|}
\hline From & To & Type & IIme & lane & Length & Speed & VPH & $\begin{array}{l}\frac{\text { Real }}{\text { Travel }} \\
\text { Time }\end{array}$ & $\begin{array}{l}\text { Delay } \\
\text { Time }\end{array}$ & PHF & $\underline{P C U}$ & $\underline{v / c}$ & Density & $\underline{\cos }$ \\
\hline 60 & 44 & UNK & 57 & 2 & 1012 & 63 & 308 & 46 & 11 & 0.95 & 182 & \begin{tabular}{|l|}
0.1 \\
\end{tabular} & 2.85 & A \\
\hline 44 & 46 & UNKK & 9 & 2 & 160 & 64 & \begin{tabular}{|l|l|}
114 \\
\end{tabular} & 7 & 2 & \begin{tabular}{|l|l|} 
\\
\end{tabular} & 67 & \begin{tabular}{|l|l|}
0.16 \\
\end{tabular} & 1.05 & $\mathrm{~A}$ \\
\hline 46 & 25 & UNK & 20 & 2 & 250 & 45 & 1968 & 11 & 9 & 0.95 & 1160 & \begin{tabular}{|l|}
0.97 \\
\end{tabular} & 25.78 & $E$ \\
\hline 25 & \begin{tabular}{|l|}
33 \\
\end{tabular} & \begin{tabular}{l|l} 
UNKK \\
\end{tabular} & 19 & 2 & 325 & 61 & 1196 & 15 & 4 & \begin{tabular}{|l|l|} 
\\
\end{tabular} & 705 & \begin{tabular}{|l|}
0.39 \\
\end{tabular} & 11.45 & $c$ \\
\hline 33 & 103 & LNK & 71 & 1 & 1050 & 53 & 1218 & 63 & 8 & 0.95 & 1436 & \begin{tabular}{|l|} 
\\
\end{tabular} & 26.97 & $E$ \\
\hline 103 & \begin{tabular}{|l|}
38 \\
\end{tabular} & ROUND & 35 & 1 & 30 & \begin{tabular}{|l|l|}
$x x$ \\
\end{tabular} & 688 & $x x$ & $x x$ & \begin{tabular}{|l|l|}
0.83 \\
\end{tabular} & $x x$ & 0.04 & $x x$ & $A$ \\
\hline 38 & 80 & UNK & 29 & 1 & 266 & 33 & 2101 & 16 & 13 & 0.95 & 2477 & 1.17 & 75.02 & $\mathrm{~F}$ \\
\hline 80 & 77 & ROUND & 30 & 1 & 50 & \begin{tabular}{ll|}
$x x$ \\
\end{tabular} & 565 & $x x$ & $x x$ & \begin{tabular}{|l|l|} 
\\
\end{tabular} & $x x$ & \begin{tabular}{|l|}
0.07 \\
\end{tabular} & $x x$ & $\mathrm{~A}$ \\
\hline 77 & \begin{tabular}{ll|}
72 \\
\end{tabular} & LINK & 37 & 1 & 332 & 32 & 2759 & 20 & 17 & 0.95 & 3252 & \begin{tabular}{l|l|}
1.29 \\
\end{tabular} & \begin{tabular}{|l|l|}
100.68 \\
\end{tabular} & $F$ \\
\hline 72 & 71 & ROUND & 8 & 2 & 154 & \begin{tabular}{ll|}
$x x$ \\
\end{tabular} & 431 & $x x$ & $x x$ & \begin{tabular}{|l|l|} 
\\
\end{tabular} & $x x$ & \begin{tabular}{|l|}
0.03 \\
\end{tabular} & $x x$ & $\mathrm{~A}$ \\
\hline 71 & 106 & UNKK & 32 & 1 & 410 & 46 & 1908 & 25 & 7 & 0.95 & 2249 & 1.14 & 48.76 & $F$ \\
\hline 106 & 110 & ROUND & 24 & 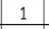 & 0 & $x x$ & 302 & $x x$ & $x x$ & \begin{tabular}{|l|l|}
0.53 \\
\end{tabular} & $x x$ & \begin{tabular}{|l|}
0.17 \\
\end{tabular} & $x x$ & $\mathrm{~A}$ \\
\hline 110 & 169 & LNNK & 33 & 2 & 447 & 48 & 1538 & 27 & 6 & \begin{tabular}{|l|l|} 
\\
\end{tabular} & 906 & \begin{tabular}{|l|}
0.54 \\
\end{tabular} & 18.58 & $D$ \\
\hline 169 & 167 & UNSIG & 46 & 2 & 30 & $x x$ & 78 & $x x$ & $x x$ & \begin{tabular}{|l|} 
\\
\end{tabular} & $x x$ & 0.36 & $x x$ & c \\
\hline
\end{tabular}

\section{6. - مرق توزيع الحجوم المرورية :}

\section{Minimum path}

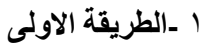

اربيل ودهوك ـ ـ وان الطرق التي تربط مدينة الموصل بهاتين

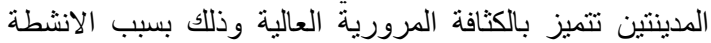

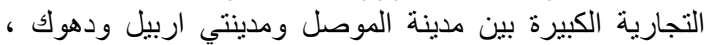

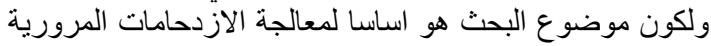

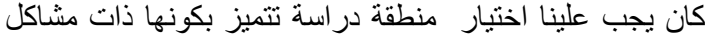

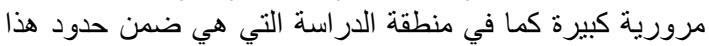

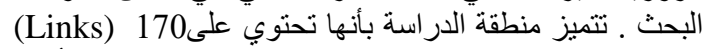

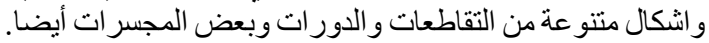
والثكل (1) يوضح منطقة الدراسة في الجانب الأيسر لمدينة

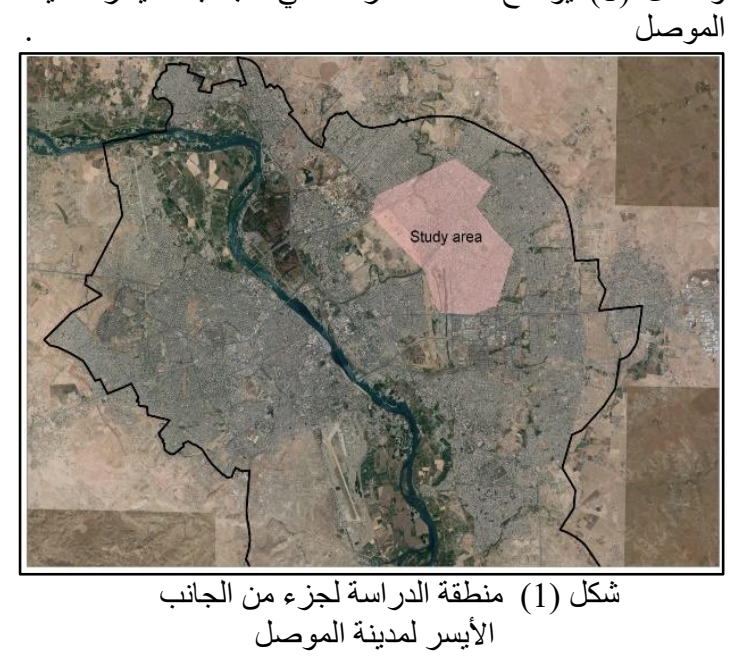

4. اعداد خر ائط المحاكاة لمنطقة الدراسة

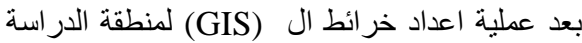

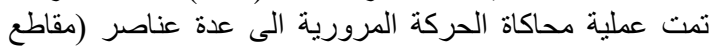

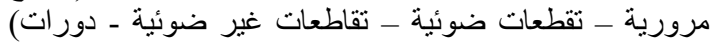

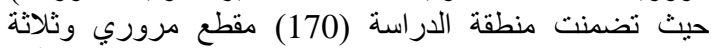

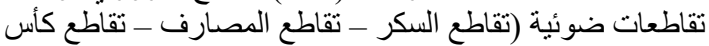

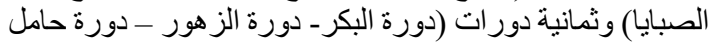

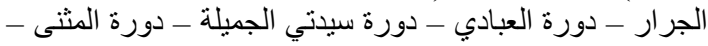

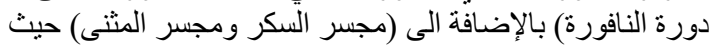

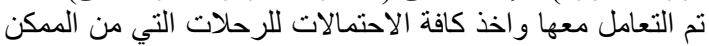

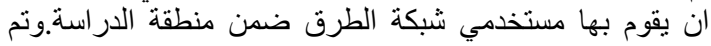

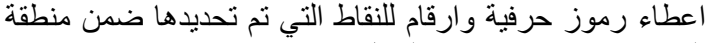
الدراسة. وكما موضح فرضي وارثام الثكل رقم (2) .

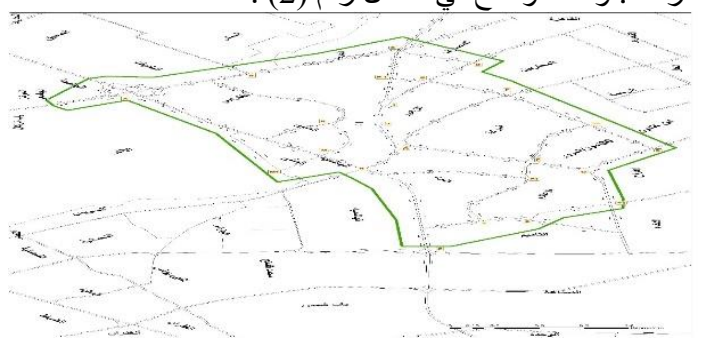

شكل(2): خارطة GIS موضح عليها المحاكاة الأولية لواقع الحال

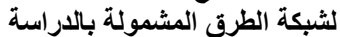

الفترات الاستراتيجية ومعدل النمو المستقبلي

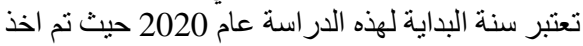

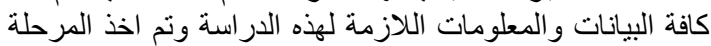
الأولى من الدر اسة من عام 2020 ولغاية عام 2025 ـ اي ان الفئ الفترة 
تمت خلال هذه المرحلة عملية مناقشة النتائج النهائية

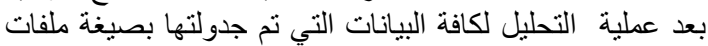

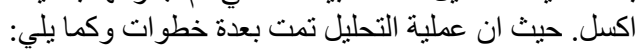

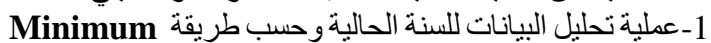
path

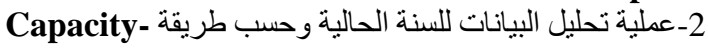
Restraint

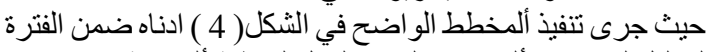
لتحليل للازدحام ألمروري لو اقع الحال لمنطقة ألدر اسة الداه

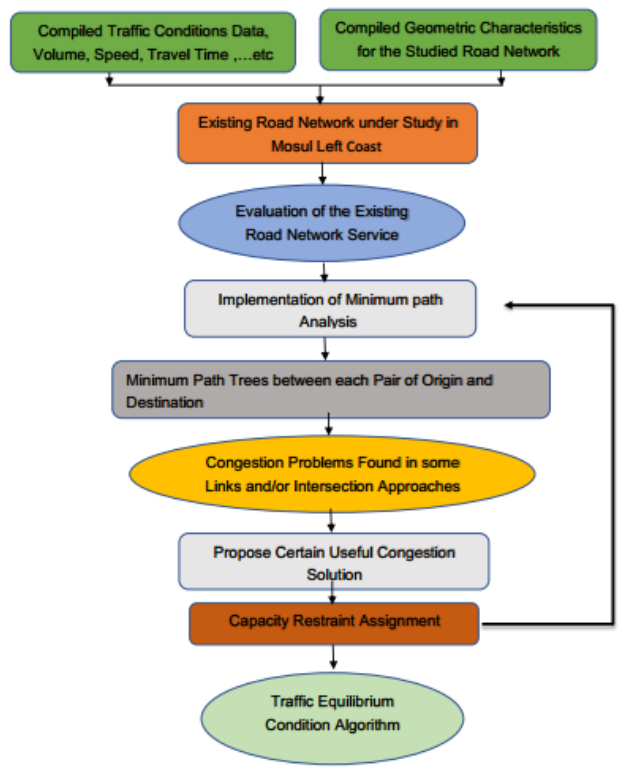

\section{شكل(4): مخطط يوضح عملية التحليل الاني أو واقع الحال لشبكة الطرق لمنطقة الدراسة التية}

تم تحليل البيانات الموضحة بالجدول (1) والتي تمثل نموذج من

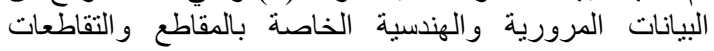

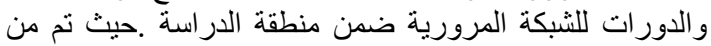

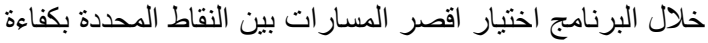

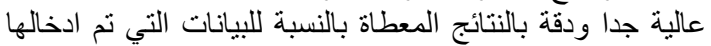

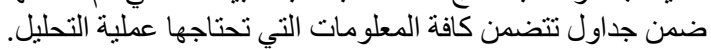

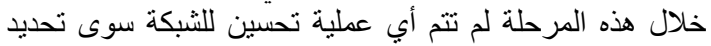

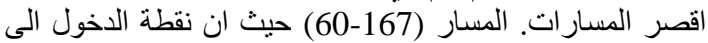

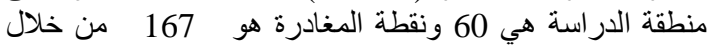

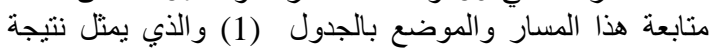

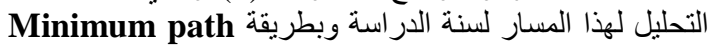

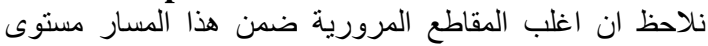

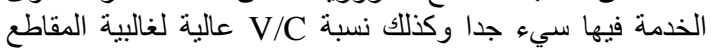

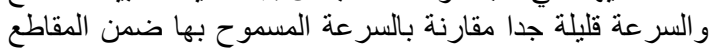

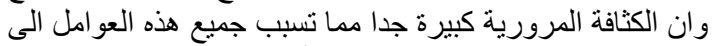

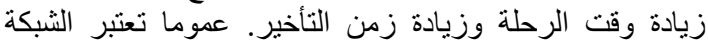

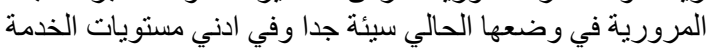
في اوقات الذروة المرورية.

\section{8. التحليل الاحصائي للنتائج}

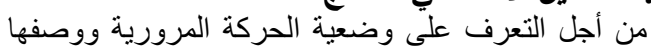
بشكل بتناسب مع طبيعة السلوك المروف التروري للمركبات في الثبكة

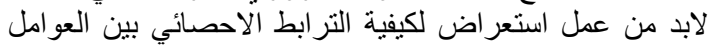

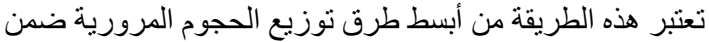

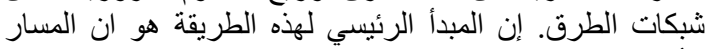

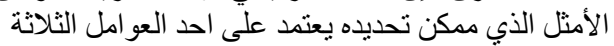

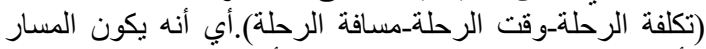

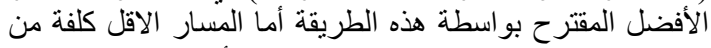

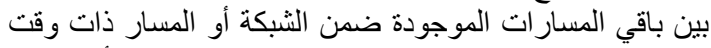

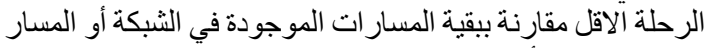

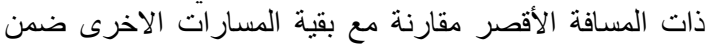

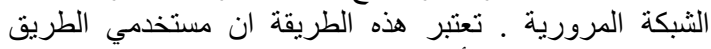

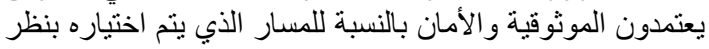

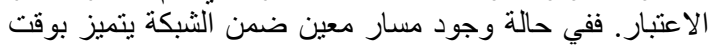

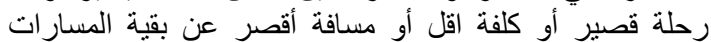

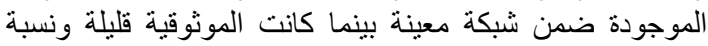

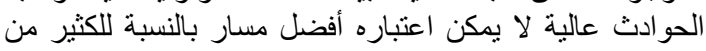
مستخدمي الشبكة .

\section{Capacity-Restraint Assignment}

r ـ ب الطريقة الثانية

تعتبر هذه الطريقة من طرق التوزيع المروري و الناتجة عن تحسين

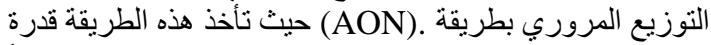

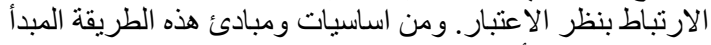

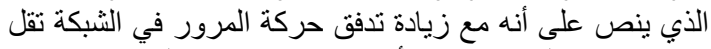

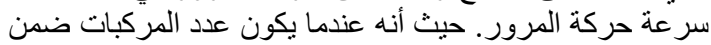

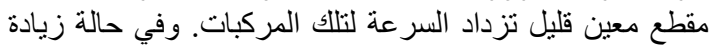

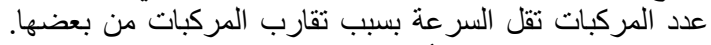

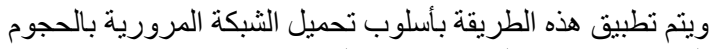

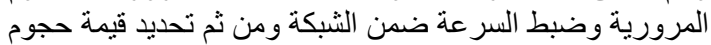

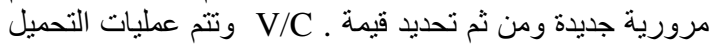

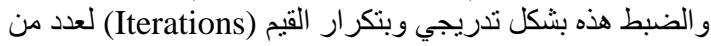

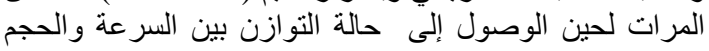

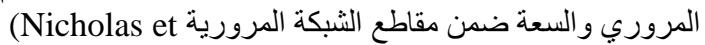
al,2009)[3].

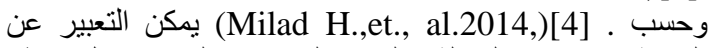

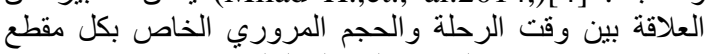

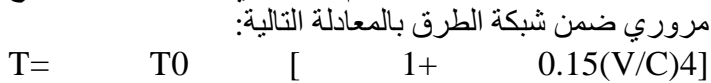
..(4-2) حيث ان: وقت الرحلة الذب يعتمد على الحجم المروري : T

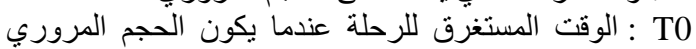
يساوي (صفر) :

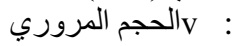
العة : :

تم تطوير العلاقة بين (السرعة الطريق الحجوم المرورية ) و المستخدمة

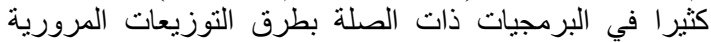

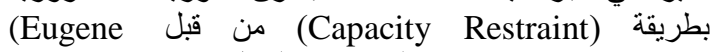
(5]V.M.,et.al,2016 )

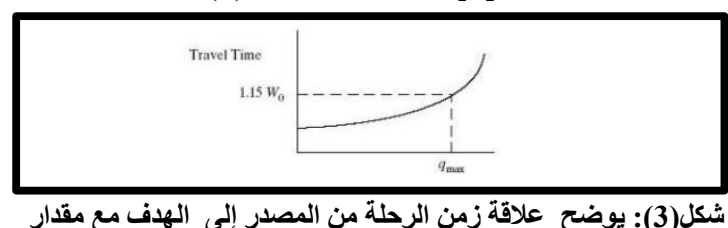

[5](Eugene V.M.,et.al,2016) الجريان المروري

7. تحليل النتائج للفترة ألحالية 
99 Mohannad R.M. Askar : Existing and Future Traffic Congestion Reduction Study......

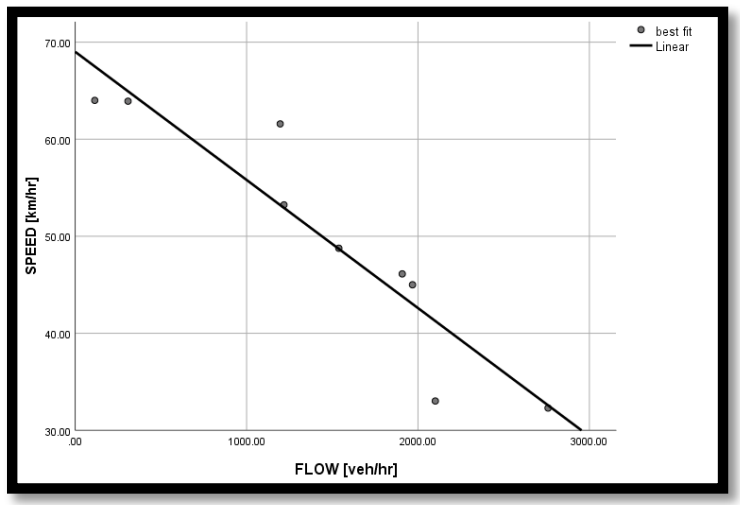

$\mathbf{y}=69.005-0.13 x$

شكل (7):العلاقة بين معاملي السرعة والجريان المروري

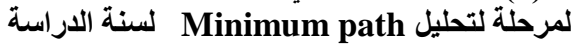

(2020-2019)

الجدول(2): مقدار الثقة في قبول او رفض العلاقات الاحصائية

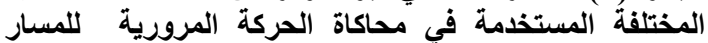

(60 - 167)

\begin{tabular}{|c|c|c|c|}
\hline$\underline{\text { Tvpe of model }}$ & $\frac{\underline{\text { Correlation }}}{\underline{\text { Coefficient R }}}$ & $\frac{\underline{\text { Correlation }}}{\underline{\text { Coefficient R }}}$ & $\begin{array}{c}\underline{\text { Sigunificant }} \\
\underline{\text { Yes, or not }}\end{array}$ \\
\hline Flow - Density & 0.915 & 0.838 & Yes \\
\hline Speed - Density & 0.896 & 0.802 & Yes \\
\hline Speed - Flow & 0.929 & 0.863 & Yes \\
\hline
\end{tabular}

مقارنة أفضل مسار بين النقتين (60-167) ومسارين اخرين Comparison the best path between (60-167) and other two paths

بعد عملية التحليل وظهور النتائج تم تحديد ثناثة مسار ات بين

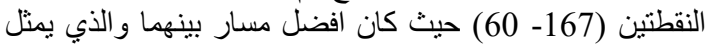

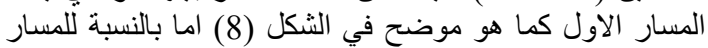

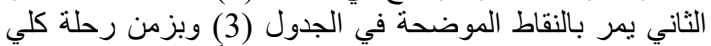

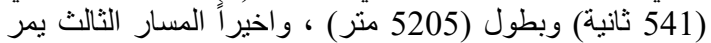

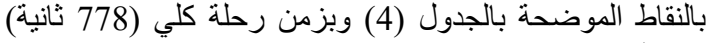
وبطول (5690 منر).
المرورية الخاصة لوصف هذا السلوك للشبكة التي تم اختيارها

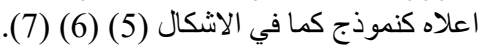

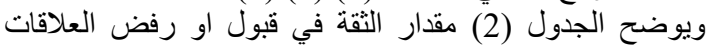

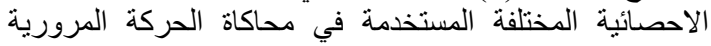
اللمسار (60 - 167)

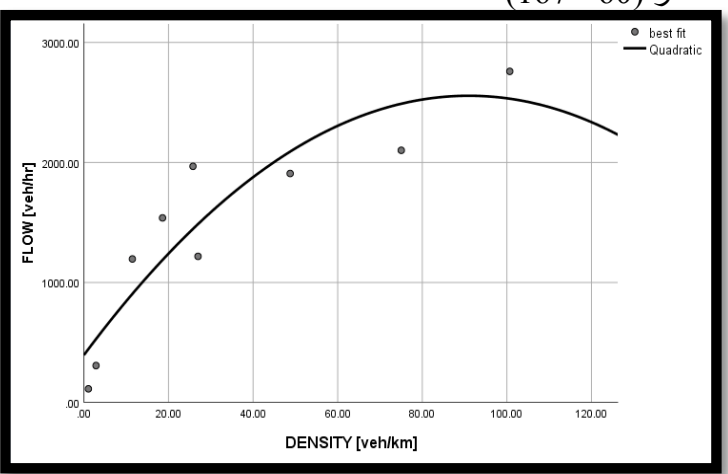

$\mathrm{y}=395.507+47.487 \mathrm{x}-0.261 \mathrm{x} 2$

شكل (5):العلاقة بين معاملي الجريان المروري والكثافة

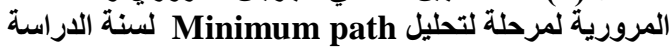
(2020-2019)

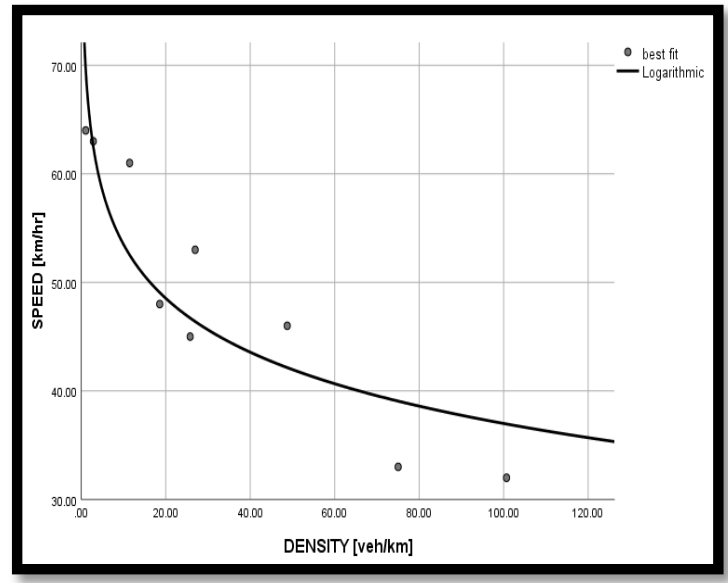

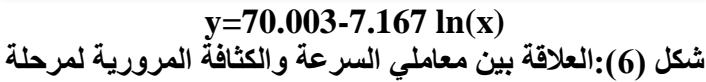
لتحليل Minimum path لسنة الدعة ولدينة (2020-2019) 
الجدول:( 4) نتائج التحليل للمسار الثالث بين النقطتين(60-167-1920)

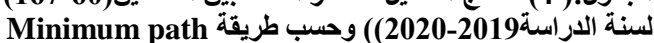

\begin{tabular}{|c|c|c|c|c|c|c|c|c|c|c|c|c|c|}
\hline 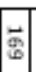 & $\vec{b}$ & $=$ & & & $\infty$ & & & $\triangle$ & & 5 & & & 霜 \\
\hline 5 & & $\overrightarrow{8}$ & & $\lambda$ & $y$ & & $\%$ & $=$ & \ & $=$ & & 12 & $\mid \vec{a}$ \\
\hline & & $\sum_{x}$ & & & $\frac{5}{5}$ & 吾 & $\frac{5}{2}$ & 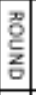 & & 哀 & & $\sum_{x} \bar{z}$ & 居 \\
\hline 5 & & 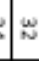 & $\infty$ & $\omega$ & 5 & & 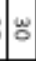 & $\overrightarrow{0}$ & & $\infty$ & & $\underset{\downarrow}{\omega}$ & 崖 \\
\hline$N$ & & - & & -1 & 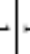 & & - & -1 & N & $\sim$ & & $\omega$ & 5 \\
\hline 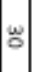 & 点 & $\stackrel{5}{\circ}$ & $\overrightarrow{5}$ & $\omega$ & 5 & & 㟧 & $\overrightarrow{0}$ & & s. & & $\stackrel{\mathrm{E}}{\mathrm{E}}$ & 臣 \\
\hline$\underset{x}{x}$ & ث) & 6 & & $\stackrel{\sim}{\sim}$ & $\times$ & & $\omega$ & $\times$ & & $\underset{\times}{\times}$ & & 血 & 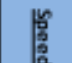 \\
\hline$\times$ & : & 8 & & 8 & $x$ & $\times$ & 8 & $\times$ & $\stackrel{0}{\circ}$ & $\mathbb{x} \mid:$ & & $\approx$ & 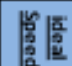 \\
\hline $\mid$ & $\overrightarrow{\mathrm{w}}$ & $\overrightarrow{\$}$ & th & 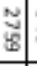 & 8 & & $\frac{\sqrt{8}}{8}$ & $\begin{array}{l}u \\
w \\
\vdots\end{array}$ & 8 & . & & Е & |ફ \\
\hline$\underset{x}{x}$ & 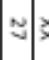 & N & & $\approx$ & $8 \mid$ & $\times$ & $\vec{\infty}$ & $\times$ & & $\underset{\times}{\times}$ & & $\approx$ & 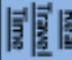 \\
\hline$\times$ & ब. & $\checkmark$ & & $\exists$ & $x \mid:$ & & $\vec{N}$ & $\times$ & 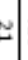 & $\underset{\times}{\times}$ & & $\overline{5}$ & 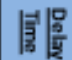 \\
\hline o & : & 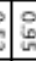 & i & 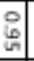 & ? & 8 & 용 & : & ? & 9 & & 过 & 胥 \\
\hline$x$ & 竞 & $\widehat{N}$ & & 5 & & & S & $\times$ & है & $\times \frac{3}{\times} \times \frac{3}{7}$ & & 串 & 㞎 \\
\hline 遂 & 9 & $=$ & 8 & $\exists$ & 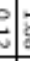 & & $\overrightarrow{\mathrm{\sigma}}$ & $\begin{array}{l}9 \\
\end{array}$ & 5 & $\begin{array}{c}0 \\
0 \\
\vdots\end{array}$ & & 过 & $\leqslant$ \\
\hline$\times$ & $\left|\begin{array}{c}\vec{\infty} \\
\vec{\omega}\end{array}\right|$ & $\hat{\infty}$ & $\times$ & d & 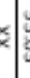 & $\times$ & 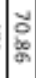 & $\times$ & $\Delta$ & $\times$ & & 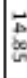 & 根 \\
\hline & $0=$ & 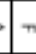 & 1 & & & & (") & 0 & & $m$ & & & 格 \\
\hline
\end{tabular}

\section{9. تحليل البيانات للفترة المستقبلية}

تمت خلال هذه المرحلة عملية مناقشة النتائج النهائية

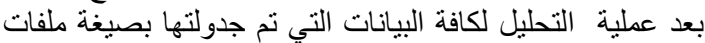

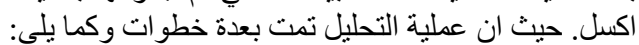

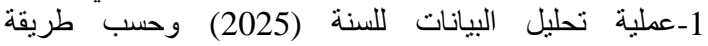

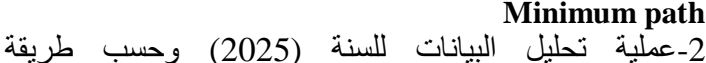

Capacity-Restraint

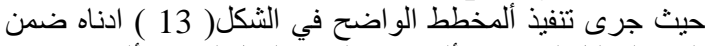

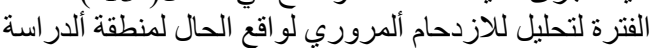

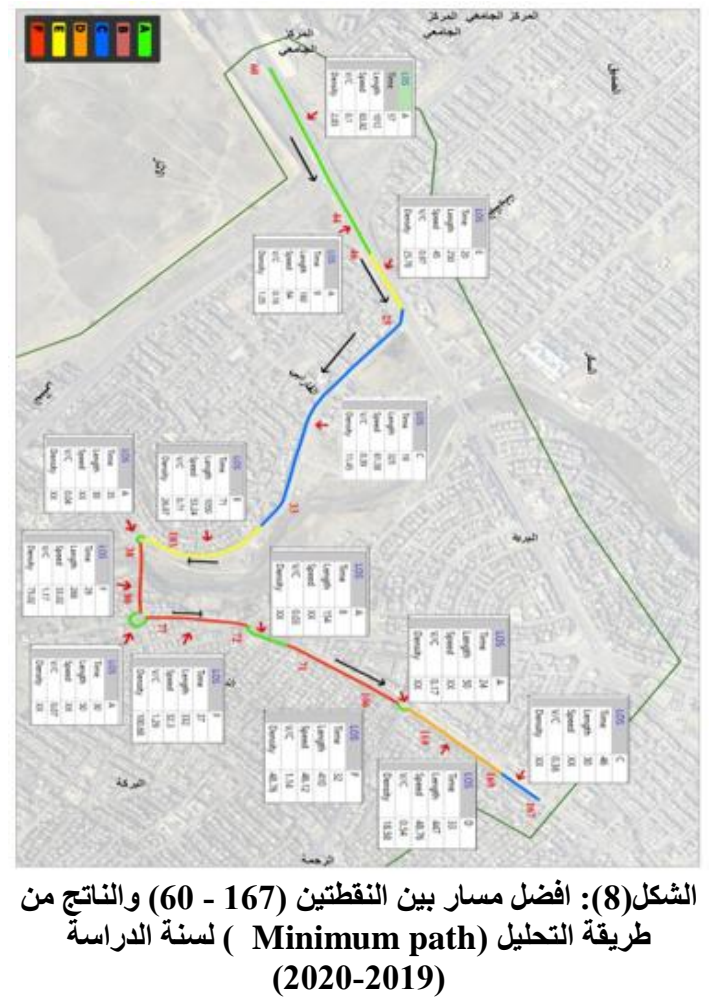

جدول:(3) نتائج التحليل للمسار الثاني بين النقطتين(2020(167-100) لسنة

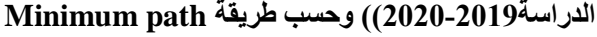

\begin{tabular}{|c|c|c|c|c|c|c|c|c|c|c|c|c|c|c|}
\hline 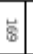 & & $\overrightarrow{8}$ & $\approx$ & & & & & $\theta$ & $\AA$ & & & & 8 & 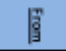 \\
\hline 잉 & & $\overrightarrow{\mathrm{s}}$ & 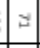 & $\approx$ & $\exists$ & 8. & & $\approx$ & $\approx$ & $\$ 5$ & & & $E$ & 10 \\
\hline 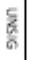 & & 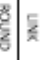 & 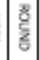 & ह & 吾 & t) & 质 & 닷 & 랏 & 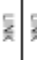 & & 氛 & 랏 & 8 \\
\hline E & & $\approx \approx$ & $=\infty$ & $\because$ & 8 & 6 & & 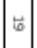 & 8 & $\approx 0$ & $\approx$ & $:$ & 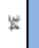 & 責 \\
\hline$\sim$ & &.+ & $\sim$ & - & + & 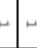 & 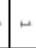 & $\sim$ & $\sim$ & $\sim$. & $\omega$ & $\sim$ & $\omega$ & 5 \\
\hline 8 & & 8 \& & $\overrightarrow{5}$ & $\ddot{*}$ & 8 & 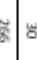 & & $\ddot{*}$ & 8 & $\mathbb{E}$ & & 8 & 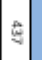 & $\sqrt{3}$ \\
\hline ๘્ર & & $\approx \neq$ & $=\times$ & $\approx$ & 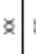 & $\approx$ & & 2 & $\Leftrightarrow$ & $\approx$ & & $\approx$ & \& & 裳 \\
\hline ×्र & & 88 & 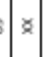 & 8 & 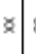 & $8: 5$ & & 8 & 8 & 8 & & 8 & 8 & 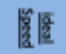 \\
\hline$\approx$ & & 8 & 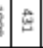 & : & s. & تै & & : & 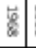 & $\frac{8}{8} 8$ & & 落 & 空 & 厘 \\
\hline 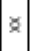 & & $\approx \approx$ & 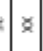 & $\approx$ & 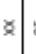 & 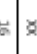 & $\mathscr{Z}$ & $\Rightarrow$ & 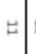 & 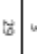 & & $\approx$ & $\approx$ & 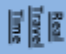 \\
\hline |x & & $\approx$. & 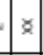 & $\forall$ & 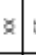 & \pm & $\infty$ & ${ }^{\star}$ & $\cdot$ & $\omega$ & & $\approx$ & 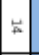 & 䝿展 \\
\hline @ & & $\%$ & : & : & 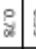 & 6 & & $\%$ & 8 & $: 8$ & & $\%$ & $\xi$ & 屋 \\
\hline ๘. & & 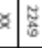 & $\approx$ & 菊 & 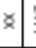 & 㱐: & 容 & : & 8 & 泀 & 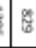 & 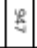 & $\approx$ & 展 \\
\hline 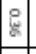 & & $\stackrel{5}{s}$ & : & 5 & : & $t$ & \% & : & 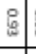 & 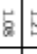 & & \& & : & 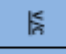 \\
\hline ×्र & 101 & 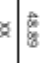 & 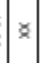 & 总 & 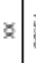 & $8=$ & & 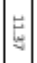 & ${ }^{2} 1$ & & & 180 & 㤐 & $\frac{3}{2}$ \\
\hline & & 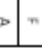 & $D$ & $\pi$ & 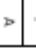 & 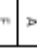 & $m$ & 1 & $m$ & $\pi$ & $=$ & 0 & \begin{tabular}{|c|} 
\\
\end{tabular} & $\sqrt{2}$ \\
\hline
\end{tabular}


101 Mohannad R.M. Askar : Existing and Future Traffic Congestion Reduction Study......

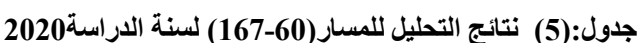

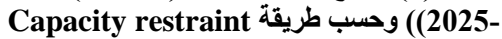

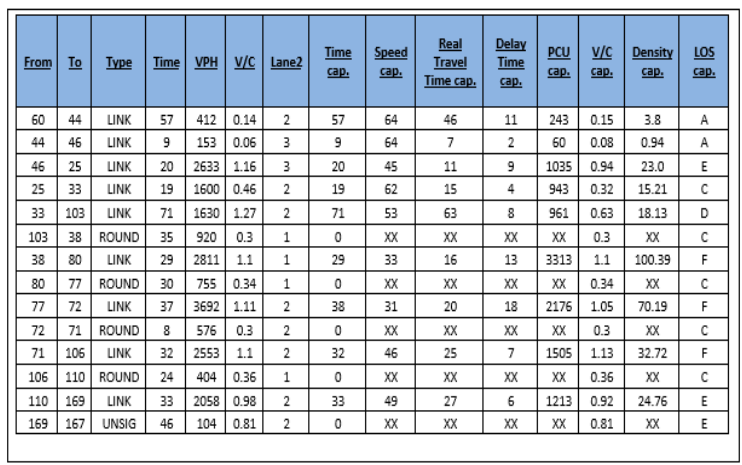

10. التحليل الاحصائي للنتائج

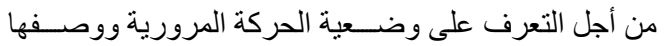

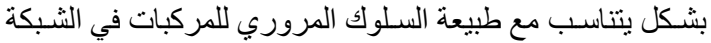

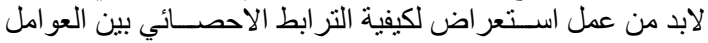

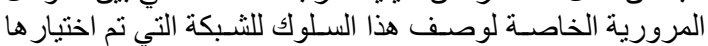

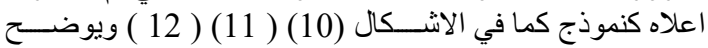

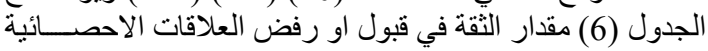

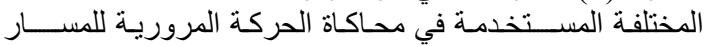

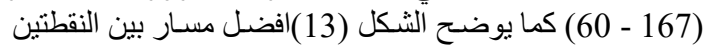

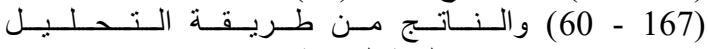
لسنة الدراسة (2025-2020) لسنة (capacity restraint)

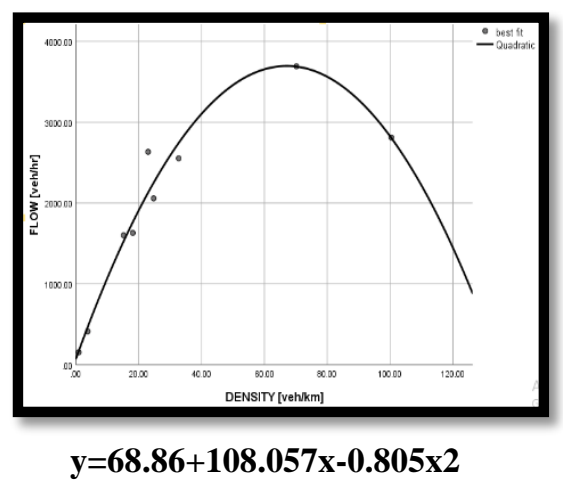

شكل (10):العلاقة بين معاملي الجريان المروري والكثافة

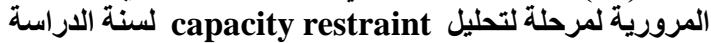

(2025-2020)

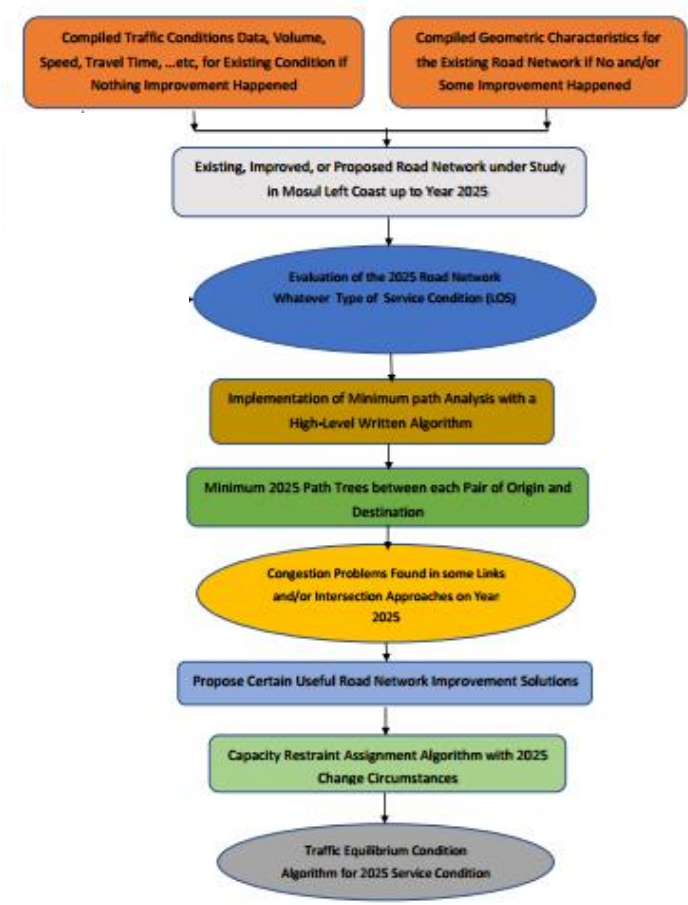

شكل(9): مخطط يوضح عملية التحليل المستقبلية لشبكة الطرق لمنطقة الاراسة (للسنة 2025)

تم تحليل البيانات الموضحة بالجدول (5) و التي تمثل نموذج من بن بالئن

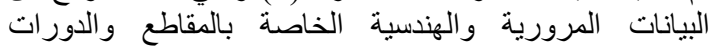

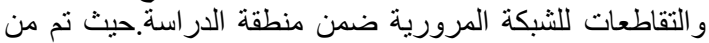

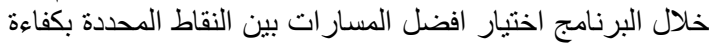

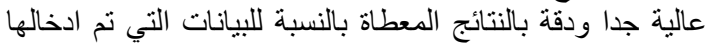

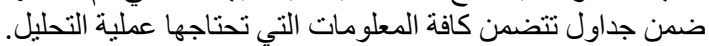

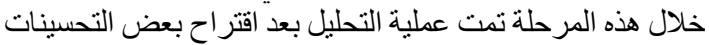

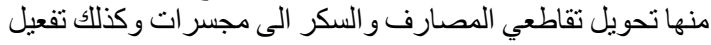

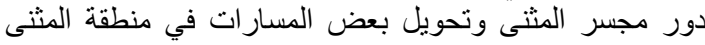

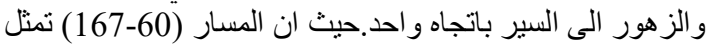

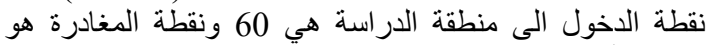

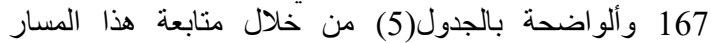
و الموضح بالجدول (5) والذولي يمثل نتيجة التحليل لهذا المسار

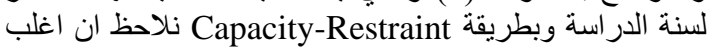
المقاطع المرورية ضمن ضيطنة هذا المسار مستوى الخدمة فيها جيدجدا

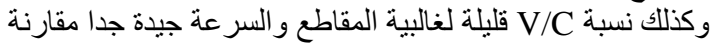

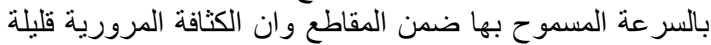

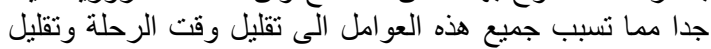

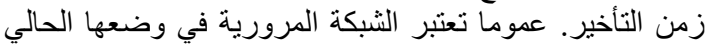
جيدة جدا وفي افضل مستويات الخدمة في اوقات ألذروة المرورية. 


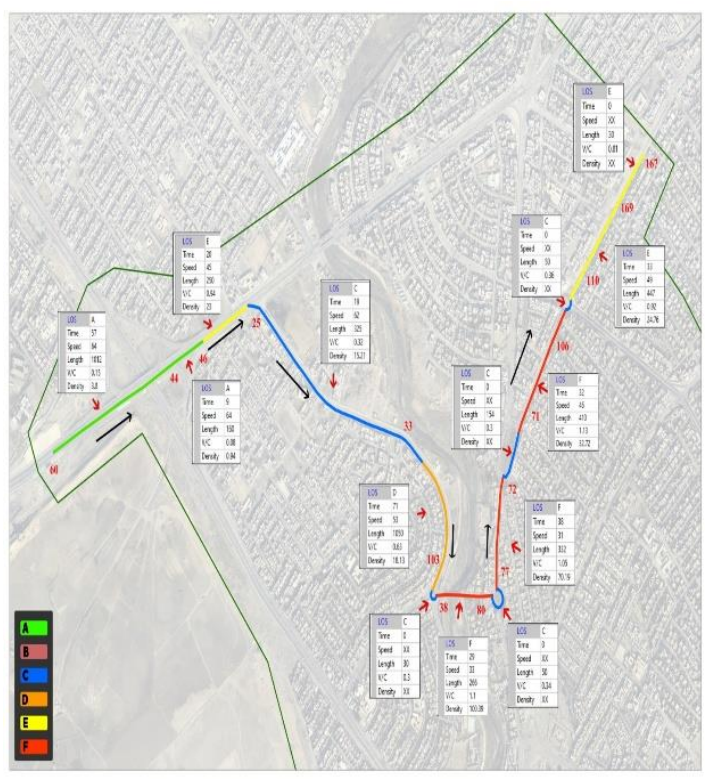

الشكل(13): افضل مسار بين النقطتين (167 - 60 (60) والناتج من طريقة التحليل (capacity restraint) لسنة (13) - الدراسة (2020-2025)

11. - 11 الاستنتاجات

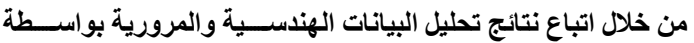

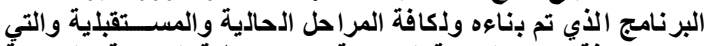

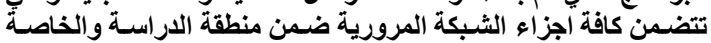
بموضوع هذه الرسالة تم التوصل إلى التى الاستنتاجات الاتية:

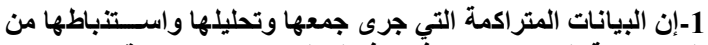

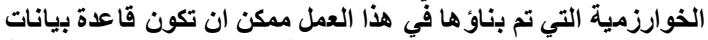

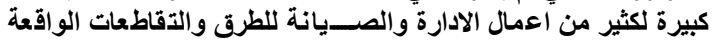
ضمنها.

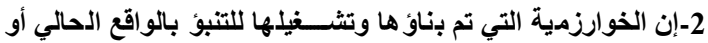

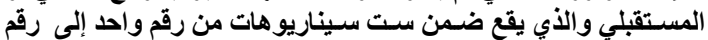

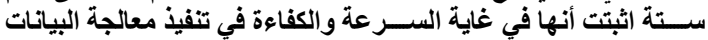
واستنباط نتائج معقولة.

3 -ان خوارزمية Minimum path جيدة في ايجاد اقصر المسارات

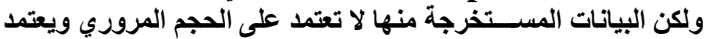

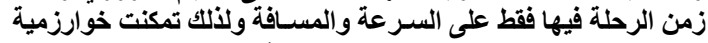

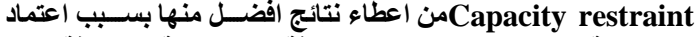

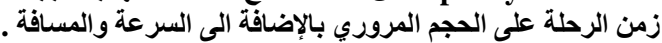

4 ـ عند اضافة مسار الى مقطع معين لا يحدث تحسن في الزمن ويحدث

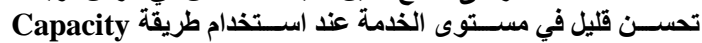

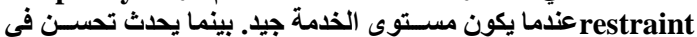

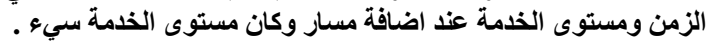

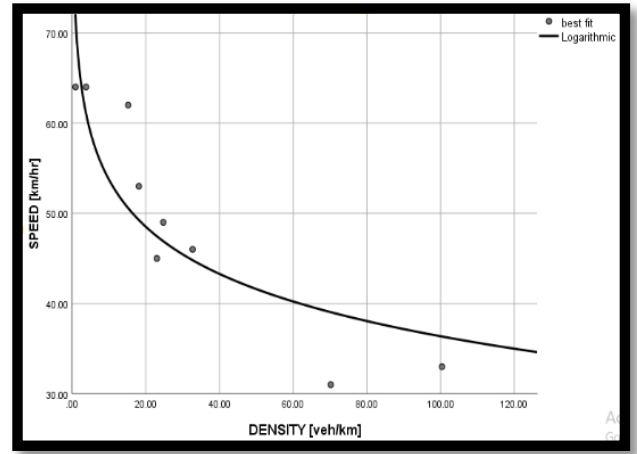

$y=71.117-7.546 \ln (x)$

شكل (11):العلاقة بين معاملي السرعة والكثافة المرورية لمرحلة تلحليل capacity restraint لسنة الدراسة (11) (2025-2020)

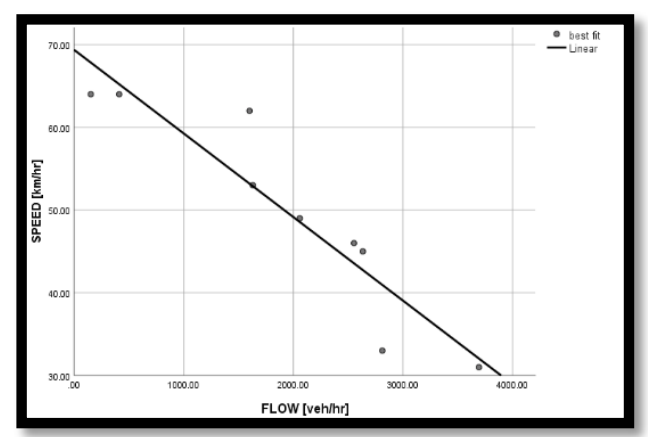

شكل (12):العلاقة بين معاملي السرعة و الجريان المروري لمرحلة

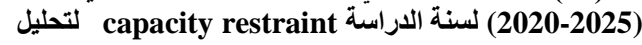

الجدول(6): مقدار الثقة في قبول او رفض العلاقات الاحصائية المختلفة المستخدمة في محاكاة الحركة المرورية للمبلة اللمسار (60 - 167)

\begin{tabular}{|c|c|c|c|}
\hline Type of model & \begin{tabular}{|l}
$\frac{\text { Correlation }}{\text { Coefficient }}$ \\
$\underline{\mathbf{R}}$
\end{tabular} & \begin{tabular}{|c|}
$\frac{\text { Correlation }}{\text { Coefficient } R}$ \\
square
\end{tabular} & $\begin{array}{l}\text { Significant } \\
\underline{\text { Yes, or not }}\end{array}$ \\
\hline Flow - Density & 0.983 & 0.966 & Yes \\
\hline Speed - Density & 0.871 & 0.759 & Yes \\
\hline Speed -Flow & 0.929 & 0.863 & Yes \\
\hline
\end{tabular}




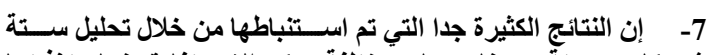

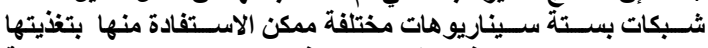

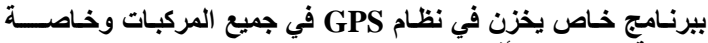
الحديثة منها لاتباع أقصر في المسارات.

[1] Arc GIS 10.7., Version.( 2016)

[2] B.Robert Smock., "A Comparative Description of a Capacity-Restrained Traffic Assignment", Detroit Area Traffic Study, Wayne State University. HRR No.6., Traffic Assignment 4 Reports, National Academy of Science, National Research Council, Publication 1102,1999;

[3] J.Nicholas Garber \& Lester A. Hoel. "Traffic and HighwayEngineering". Fourth Edition University of Virginia; (2009).

[4] H.Milad, Zahra S., Majid S. (Probit Based Traffic Assignment A Comparative Study between Link Based Simulation Algorithm and Path Based Assignment and Generalization to Random Coefficient Approach). International Journal of Transportation Engineering; (2014).,

[5] V.M.Eugene , et al.,'Impact of Optimally Minimizing Delay Times on Safety at Signalized Intersections in Urban Areas, Case Study:The City of Virginia Beach",International Journal of Transportation Engineering Vol.3/No,4?Spring 2016.

[6] Microsoft Corporation. 'Statistical Package for Social Science' 'Word Program, Version-24, 2018;

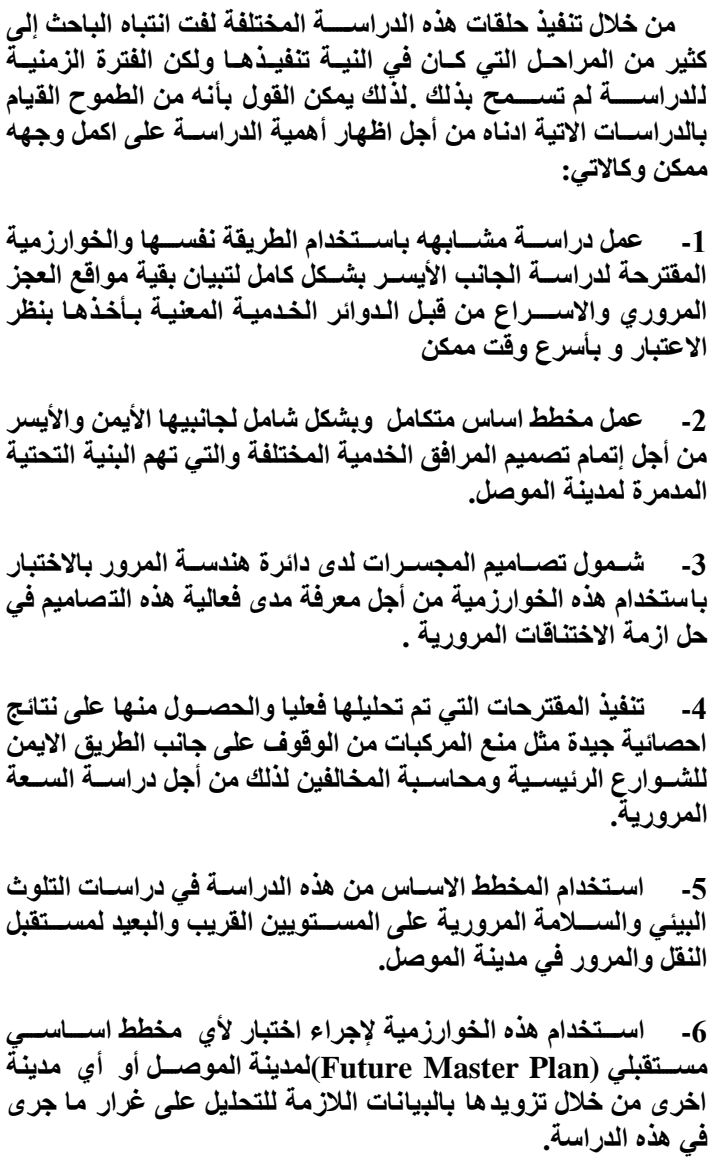

\title{
Existing and Future Traffic Congestion Reduction Study in Mosul Left Coast Using an Electronic Algorithm
}

\author{
Mohannad R.M. Askar \\ civilmohanadtraf2018@gmail.com
}

\author{
Abdulkhalik M. Al-Taei \\ abdelkhaleq@uomosul.edu.iq
}

Civil Engineering Department, Collage of Engineering, University of Mosul

\begin{abstract}
$\underline{\text { Abstract }}$
Traffic mapping is the fourth, final, and very important step of the urban transport planning process. the algorithm that was developed using several programs. Such as the Geographic Information Systems (GIS) program, the Digextra algorithm, the Python program, in addition to the (SIDRA-8-2018) program, which is a program specialized in engineering work for methods to determine the shortest path within the selected network during the present time in addition to future strategic periods.Three future periods have been determined to test the road network load with traffic volumes, determine the locations of defects that will occur now and, in the future, and try to find solutions and alternatives that ensure the flow of traffic within this network. Each strategy period included the growth of traffic demand for a period of five years, starting from 2020 until the target year 2025. During these five years, traffic demand is supposed to increase by 6\% annually. The results obtained from this study were represented by different plans to address the traffic problems that occur in the different areas of the road network. Those areas were identified and different scenarios and solutions would be required to regulate the traffic for the strategic periods.
\end{abstract}

Keywords:

Traffic Assignment; Mosul City; Level of Service; Capacity Restraint. 